\title{
Theoretical and numerical considerations on Bratu-type problems
}

\author{
Adrian Petruşel, Ioan A. Rus and Marcel Adrian Şerban
}

Dedicated to Professor Gheorghe Moroşanu on the occasion of his 70th anniversary.

\begin{abstract}
In this paper we present an heuristic introduction to Bratu problem and we give some variants of Bratu's theorem (G. Bratu, Sur les équations intégrales non linéaires, Bulletin Soc. Math. France, 42(1914), 113-142). Using the positivity of Green's function, the monotone iterations technique and the contraction principle, some generalizations of Bratu's result are also given. $\mathrm{Nu}-$ merical aspects are also considered.
\end{abstract}

Mathematics Subject Classification (2010): 34B18, 47H10, 65R20, 34B27, 45G10, $35 \mathrm{~K} 58$.

Keywords: Bratu problem, Bratu theorem, Bratu-type equation, Cauchy problem, Nicoletti problem, boundary value problem, Green function, integral equations with exponential nonlinearity, numerical aspects of Bratu's problem.

\section{Introduction}

The classical Bratu problem is the following boundary value problem

$$
\left\{\begin{array}{l}
-y^{\prime \prime}(x)=\lambda e^{y(x)}, x \in[0,1] \\
y(0)=0, y(1)=0
\end{array}\right.
$$

where $\lambda>0$ is a parameter.

Bratu's problem has both theoretical and applicative relevance.

It was proved that Bratu's problem in one-dimensional planar coordinates has analytical solution in the following form:

$$
y(x)=-2 \log \left(\frac{\cosh \left[\left(x-\frac{1}{2}\right) \frac{\theta}{2}\right]}{\cosh \frac{\theta}{4}}\right),
$$

where $\theta$ is the solution of

$$
\theta=\sqrt{2 \lambda} \cosh \frac{\theta}{4}
$$


Notice that $y$ has the maximum value (denoted by $\mu$ ) at $x=\frac{1}{2}$ and there is an analytical expression between $\mu$ and $\lambda$ discovered by Liouville in 1853, see [37]. Moreover, Bratu's problem has at most two solutions and the distribution of the solutions depends on a critical value of $\lambda$, denoted by $\lambda_{c}$. The critical value $\lambda_{c}$ satisfies the equation

$$
1=\frac{1}{4} \sqrt{2 \lambda} \sinh \frac{\theta}{4}
$$

and it was approximated as $\lambda_{c} \approx 3.513830719$, see, e.g. [9]. More precisely, if $0<\lambda<$ $\lambda_{c}$ then (1.1) has two solutions, if $\lambda=\lambda_{c}$ there is one solution for (1.1), while for $\lambda>\lambda_{c}$ there is no solution for Bratu's problem.

Bratu's problem governs several important real life problems, such as the fuel ignition model in the thermal combustion theory, the model of thermal reaction process, the Chandrasekhar model related to the expansion of the universe and to some relativity theory models and it is connected to models from chemical reaction theory, radiative heat transfer theory and nanotechnologies (see [23], [19], [20], [1], [15], $[22], \ldots)$.

In the last two decades, many published papers have focused on solving (1.1) by analytical (e.g., Adomian decomposition method, homotopy analysis method, variational iteration methods, Laplace transform decomposition method or differential transformation method) and numerical (e.g., B-spline method, the finite difference method, weighted residual method, the shooting method, multigrid-based methods, the Sinc-Galerkin method, collocation methods based on B-spline basis functions, Bessel collocation method) methods, see [13], [14], [15], [51], [52], [7], [8], [9], [16], [17], [29], [32], [35],...

An extension of Bratu's problem is the following boundary value problem, socalled Liouville-Bratu-Gelfand problem (see [26], [21], [27], [28], [30], [35], [41]) :

$$
\left\{\begin{array}{l}
-\Delta u(x)=\lambda e^{u(x)}, x \in \Omega \\
u(x)=0, x \in \partial \Omega
\end{array}\right.
$$

where $\lambda>0$ is a parameter and $\Omega \subset \mathbb{R}^{n}$ is a bounded domain.

The aim of our paper is to we give some variants of a Bratu's theorem (G. Bratu, Sur les équations intégrales non linéaires, Bulletin de la Soc. Math. France, 42(1914), 113-142) using the positivity of Green's function, monotone iteration technique and the contraction principle. Some generalizations of Bratu's result are also given.

The structure of the paper is the following one:

1. Introduction

2. Preliminaries

3. Heuristic considerations on particular solutions of Bratu equation

4. Some variants of Bratu theorem

5. Bratu-type problems

6. Other generalizations

7. Numerical aspects of Bratu-type problem.

The Reference list will conclude the paper. 


\section{Preliminaries}

\subsection{Linear two point boundary value problem}

Let $L_{0}:=-\frac{d^{2}}{d x^{2}}+p(x) \frac{d}{d x}$ and $L:=L_{0}+q(x)$, where $p, q \in C[a, b]$. We consider the following two-point boundary value problem

$$
\begin{gathered}
L(u)=f \\
l_{1}(y ; a):=a_{10} y(a)-a_{11} y^{\prime}(a)=r_{1} \\
l_{2}(y ; b):=a_{20} y(b)+a_{21} y^{\prime}(b)=r_{2}
\end{gathered}
$$

where $f \in C[a, b], a_{i j} \geq 0, i=1,2, j=0,1, a_{10} \cdot a_{20}>0$ and $r_{1}, r_{2} \in \mathbb{R}$.

It is well known that if $q(x) \geq 0$, for $x \in[a, b]$, then the Green function for this problem exists and is positive. However, the assumption $q(x) \geq 0$ is not a necessary condition for the positivity of the Green's function. Moreover, we have the following theorem of equivalent statements concerning the positivity of Green's function.

Theorem 2.1. (I.A. Rus [47]) The following statements are equivalent:

(i) There exists a function $v \in C^{2}(] a, b[) \cap C^{1}[a, b]$ such that: $v>0$ on $[a, b]$, $\left(L_{0}+q\right)(v)>0$ on $[a, b], l_{1}(y ; a)>0$ and $l_{2}(y ; b)>0$.

(ii) The following implication holds:

$$
y \in C^{2}(] a, b[) \cap C^{1}[a, b],\left(L_{0}+q_{1}\right)(y)=0, l_{1}(y ; a)=0, l_{2}(y ; b)=0 \Rightarrow y=0,
$$

for each $q_{1} \in C[a, b]$ with $q_{1}(x) \geq q$.

(iii) The following implication holds:

$$
y \in C^{2}(] c, d[) \cap C^{1}[c, d],\left(L_{0}+q\right)(y)=0, l_{1}(y ; c)=0, l_{2}(y ; d)=0 \Rightarrow y=0,
$$

for each $[c, d] \subset[a, b]$.

(iv) There exists the Green function $G(x, s)$, corresponding to problem (2.1), (2.2), (2.3), and $G(x, s) \geq 0, \forall x, s \in[a, b]$.

(v) The first eigenvalue of the Sturm-Liouville problem

$$
\begin{gathered}
\left(L_{0}+q\right)(y)=\lambda y, \\
l_{1}(y ; a)=0, l_{2}(y ; b)=0,
\end{gathered}
$$

is positive.

By definition, we have the strong uniqueness property for the problem (2.1), (2.2), (2.3) if one (i.e., all) of the statements, in the above Theorem 2.1, is a theorem. In this case, we call the interval $[a, b]$, a strong uniqueness interval.

In many results on boundary value problems, the condition $q(x) \geq 0$ appears. The problem is in which of them we can put a strong uniqueness condition instead of $q(x) \geq 0$ condition ?

In deep connection with this problem is the following notion. Let us consider the second order linear differential equation

$$
L y:=-y^{\prime \prime}+p y^{\prime}+q y=0, \text { for } x \in[a, b] \text {, where } p, q \in C[a, b] .
$$

We suppose that $[a, b]$ is not a strong uniqueness interval with respect to $\left(L, l_{1}, l_{2}\right)$, where $l_{1}(y)(a)=y(a)$ and $l_{2}(y)(b)=y(b)$. 
By definition, an interval $[\alpha, \beta[\subset[a, b]$ is a maximum strong uniqueness interval in $[a, b]$ if $[\alpha, \beta]$ is not a uniqueness interval and each interval $[c, d] \subset[\alpha, \beta[$ is a strong uniqueness interval.

Let $h(p, q):=\min \{\beta-\alpha:[\alpha, \beta[$ is a maximum strong uniqueness interval in $[a, b]\}$. It is clear that $h(p, q)>0$. An interesting problem is to give estimates for $h(p, q)$ in terms of $p$ and $q$, see [41], [42], [4],[5], [47], [18], [46] (pp. 99-112).

Remark 2.2. For the Green function technique in nonlinear boundary value problems, see [5], [24], [44], [45], [48], [4], [11], [30], [36], [40], [42], [38],..

\subsection{Saturated contraction principle}

In our paper, we shall use the following variant of the contraction principle.

Theorem 2.3. [49] Let $(X, d)$ be a complete metric space and $f: X \rightarrow X$ be an l-contraction. Then we have:

(i) There exists $x^{*} \in X$ such that

$$
F_{f^{n}}=\left\{x^{*}\right\}, \forall n \in \mathbb{N}^{*} .
$$

(ii) For all $x \in X, f^{n}(x) \rightarrow x^{*}$ as $n \rightarrow \infty$.

(iii) $d\left(x, x^{*}\right) \leq \psi(d(x, f(x))), \forall x \in X$, where $\psi(t)=\frac{t}{1-l}, t \geq 0$.

(iv) If $\left\{y_{n}\right\}_{n \in \mathbb{N}}$ is a sequence in $X$ such that

$$
d\left(y_{n}, f\left(y_{n}\right)\right) \rightarrow 0 \text { as } n \rightarrow \infty
$$

then $y_{n} \rightarrow x^{*}$ as $n \rightarrow \infty$.

(v) If $\left\{y_{n}\right\}_{n \in \mathbb{N}}$ is a sequence in $X$ such that

$$
d\left(y_{n+1}, f\left(y_{n}\right)\right) \rightarrow 0 \text { as } n \rightarrow \infty
$$

then $y_{n} \rightarrow x^{*}$ as $n \rightarrow \infty$.

\subsection{Fixed point of increasing operators}

In this section, as a tool for the monotone iteration technique, two fixed point theorems for increasing operators on an ordered Banach space $(\mathbb{B},+, \mathbb{R}, \leq)$ are presented.

Theorem 2.4. [2] Let $(\mathbb{B},+, \mathbb{R}, \leq)$ be an ordered Banach space and $X \subset \mathbb{B}$ be an order convex subset of $\mathbb{B}$. Let $f: X \rightarrow \mathbb{B}$ be an operator. We suppose that:

(1) $f$ is increasing and continuous;

(2) $f$ is relatively compact on every order interval in $X$;

(3) there exist $\bar{x}, \widehat{x} \in X$ with $\bar{x}<\widehat{x}$ such that $\bar{x} \leq f(\bar{x})$ and $f(\widehat{x}) \leq \widehat{x}$. Then:

(a) f has a minimum and a maximum fixed point in $[\bar{x}, \widehat{x}]$. Moreover, we have

$$
x_{\min }=\lim _{n \rightarrow \infty} f^{n}(\bar{x}) \text { and } x_{\max }=\lim _{n \rightarrow \infty} f(\widehat{x}) .
$$

(b) If, additionally, $x_{\min } \geq x_{\max }$, then $\left.f\right|_{[\bar{x}, \widehat{x}]}$ is a PO. 
Theorem 2.5. Let $(\mathbb{B},+, \mathbb{R},\|\cdot\|, \leq)$ be an ordered Banach space and

$$
P:=\{x \in \mathbb{B} \mid x \geq 0\} .
$$

Let $f, g: P \rightarrow P$ be two operators. We suppose that:

(i) $f$ and $g$ are increasing and continuous;

(ii) $\overline{f([0, x])}$ and $\overline{g([0, x])}$ are compact subset for each $x \in P$;

(iii) $f \leq g$;

(iv) $F_{g}=\left\{x^{*}\right\}$.

Then:

(1) the interval $\left[0, x^{*}\right]$ is invariant for $f$ and $g$;

(2) $g:\left[0, x^{*}\right] \rightarrow\left[0, x^{*}\right]$ is a Picard operator;

(3) $\left\{f^{n}(0)\right\}_{n \in \mathbb{N}}$ converges to the minimum fixed point of $f$ in $\left[0, x^{*}\right]$ and $\left\{f^{n}\left(x^{*}\right)\right\}$ converges to the maximum fixed point of $f$ in $\left[0, x^{*}\right]$;

(4) if $f$ has a unique fixed point in $\left[0, x^{*}\right]$, then $f:\left[0, x^{*}\right] \rightarrow\left[0, x^{*}\right]$ is a Picard operator.

Proof. (1) The fact that the interval $\left[0, x^{*}\right]$ is invariant with respect to $g$ follows immediately by (i) and (iv). Let $x \in\left[0, x^{*}\right]$. Then $0 \leq x \leq x^{*}$. By (i) and (iii) we have

$$
0 \leq f(0) \leq f(x) \leq f\left(x^{*}\right) \leq g\left(x^{*}\right)=x^{*} .
$$

Thus, $\left[0, x^{*}\right]$ is invariant with respect to $f$.

(2) Take any $x \in\left[0, x^{*}\right]$. Then, by (i) and (iii), we have, for any $n \in \mathbb{N}^{*}$, that

$$
0 \leq g^{n}(x) \leq x^{*} .
$$

Consequently, the sequence $\left\{g^{n}(x)\right\}_{n \in \mathbb{N}}$ is contained in the compact set $\overline{g([0, x])}$, and thus, it has at least one limit point. By induction, it is easily seen that the sequence $\left\{g^{n}(0)\right\}_{n \in \mathbb{N}}$ is increasing. This implies that it has exactly one limit point and that the whole sequence converges to this point. Since $g$ is continuous, $\left\{g^{n}(0)\right\}_{n \in \mathbb{N}}$ converges to $x^{*}$. Thus, for any $x \in\left[0, x^{*}\right]$, we have that

$$
g^{n}(0) \leq g^{n}(x) \leq x^{*}, \text { for any } n \in \mathbb{N} .
$$

By passing to the limit we get the desired conclusion.

(3) The third conclusion follows by Theorem 2.4 (a).

(4) The last conclusion follows by Theorem 2.4 (b).

Remark 2.6. For the fixed point theory in ordered sets and ordered Banach spaces see [2], [3], [33], [25], [39], [50],..

\section{Heuristic considerations on particular solutions of Bratu equation}

Let us consider Bratu's equation

$$
-y^{\prime \prime}=\lambda e^{y}, \lambda>0 .
$$

We start this section with some remarks on the solutions $y \in C^{2}(\mathbb{R})$ of this equation.

Remark 3.1. If $y$ is a solution of $\left(B_{\lambda}\right)$, then $y$ is a strictly concave function. This implies that $y^{\prime}$ is a strictly decreasing function. 
Remark 3.2. If $y$ is a solution of $\left(B_{\lambda}\right)$, then:

(1) $y(x+c), x \in \mathbb{R}$ is a solution of $\left(B_{\lambda}\right), \forall c \in \mathbb{R}$;

(2) $y(-x+c), x \in \mathbb{R}$ is a solution of $\left(B_{\lambda}\right), \forall c \in \mathbb{R}$;

(3) $c+y\left(e^{\frac{c}{2}} x\right), x \in \mathbb{R}$ is a solution of $\left(B_{\lambda}\right), \forall c \in \mathbb{R}$.

Remark 3.3. Let $y$ be a solution of $\left(B_{\lambda}\right)$ such that there exists $x_{0} \in \mathbb{R}$, with $y^{\prime}\left(x_{0}\right)=0$. Let $z(x)=y\left(2 x_{0}-x\right)$. We observe that: $z\left(x_{0}\right)=y\left(x_{0}\right)$ and $z^{\prime}\left(x_{0}\right)=-y^{\prime}\left(x_{0}\right)=0$. By the uniqueness of the solution of Cauchy problem, we have that $y(x)=z(x), \forall x \in \mathbb{R}$, i.e., $y(x)=y\left(2 x_{0}-x\right), \forall x \in \mathbb{R}$. From this, it follows that $y\left(x_{0}-x\right)=y\left(x+x_{0}\right)$, $\forall x \in \mathbb{R}$, i.e., the graphic of $y$ is symmetric with respect to the line, $x=x_{0}$.

Now let us make the change of the function $y$, by $e^{y}=\frac{1}{u^{2}}$. Then for $u$ we have the equation

$$
2\left(u^{\prime \prime} u-u^{\prime 2}\right)=\lambda
$$

If $u$ is such that $u^{\prime \prime}=u$ and $u^{2}-u^{\prime 2}=1$, then $y=\ln \frac{1}{u^{2}}$ is a solution of $\left(B_{2}\right)$. Such a function $u$ is, for example, $u(x)=\cosh (x)$. Therefore, the function $y(x)=-2 \ln (\cosh x), x \in \mathbb{R}$ is a solution of $\left(B_{2}\right)$.

In order to find a solution for $\left(B_{\lambda}\right)$, let us try with

$$
y(x)=-2 \ln \left(c_{1} \cosh c_{2} x\right), x \in \mathbb{R}, c_{1} \in \mathbb{R}_{+}^{*}, c_{2} \in \mathbb{R} .
$$

Such a function is a solution of $\left(B_{\lambda}\right)$, if $c_{1} c_{2}=\sqrt{\frac{\lambda}{2}}$. By Remark 3.2(1), if $c_{1} c_{2}=\sqrt{\frac{\lambda}{2}}$, the function

$$
y(x)=-2 \ln \left[c_{1} \cosh \left(c_{2}\left(x-x_{0}\right)+c_{3}\right)\right], x, x_{0}, c_{3} \in \mathbb{R}, c_{1}, c_{2} \in \mathbb{R}^{*}
$$

is a solution of $\left(B_{\lambda}\right)$.

Now we shall prove that, for each $x_{0} \in \mathbb{R}$, this is the general solution of $\left(B_{\lambda}\right)$. For to do this, let us consider the Cauchy problem

$$
-y^{\prime \prime}=\lambda e^{y}, y\left(x_{0}\right)=y_{0}, y^{\prime}\left(x_{0}\right)=y_{0}^{\prime}, x_{0}, y_{0} \in \mathbb{R} .
$$

From the Cauchy problem, we have for $c_{1}, c_{2}, c_{3}$, the following system of equations

$$
\left\{\begin{array}{l}
c_{1} c_{2}=\sqrt{\frac{\lambda}{2}} \\
c_{1} \cosh c_{3}=e^{-\frac{y_{0}}{2}} \\
c_{2} \tanh c_{3}=-\frac{y_{0}^{\prime}}{2}
\end{array}\right.
$$

Since this system has a unique solution, the conclusion is obvious. Moreover, we have the following result.

Theorem 3.4. The Cauchy problem for $\left(B_{\lambda}\right)$ has a unique saturated solution defined on $\mathbb{R}$. 
From this theorem it follows that:

If $y \in C^{2}[0, b]$ or $y \in C^{2}(] 0, b[) \cap C[0, b]$ is a solution of Bratu problem

$$
-y^{\prime \prime}=\lambda e^{y}, \lambda>0, y(0)=0, y(b)=0, b>0
$$

then there exists a unique solution $\widetilde{y} \in C^{\infty}(\mathbb{R})$ of $\left(B_{\lambda}\right)$ such that $\left.\widetilde{y}\right|_{[0, b]}=y$.

\section{Some variants of Bratu theorem}

We start by considering the following problems (for $\lambda>0$ ):

\section{Bratu problem:}

$$
-y^{\prime \prime}=\lambda e^{y}, y(0)=0, y(b)=0, b>0
$$

Gelfand problem:

$$
-y^{\prime \prime}=\lambda e^{y}, y(-a)=y(a)=0, a>0
$$

\section{Cauchy problem:}

$$
-y^{\prime \prime}=\lambda e^{y}, y(0)=0, y^{\prime}(0)=\mu>0
$$

\section{Nicoletti problem:}

$$
-y^{\prime \prime}=\lambda e^{y}, y(0)=0, y\left(x_{0}\right)=a, x_{0}>0
$$

For the problem $\left(B_{\lambda, b}\right)$ the following result is well known.

Bratu Theorem. ([10], [12]) For each $\lambda>0$, there exists $b^{*}(\lambda)>0$ such that:

(1) for $0<b<b^{*}(\lambda)$, the problem $\left(B_{\lambda, b}\right)$ has two solutions;

(2) the problem $\left(B_{\lambda}, b^{*}(\lambda)\right)$ has a unique solution;

(3) for $b>b^{*}(\lambda)$, the problem $\left(B_{\lambda, b}\right)$ has no solution.

For each $b>0$, there exists $\lambda^{*}(b)$ such that:

$\left(1^{\prime}\right)$ for $0<\lambda<\lambda^{*}(b)$, the problem $\left(B_{\lambda, b}\right)$ has two solutions;

$\left(2^{\prime}\right)$ the problem $\left(B_{\lambda^{*}(b), b}\right)$ has a unique solution;

$\left(3^{\prime}\right)$ for $\lambda>\lambda^{*}(b)$, the problem $\left(B_{\lambda, b}\right)$ has no solutions.

There exist some deep relations between the problems $\left(B_{\lambda, b}\right),\left(G_{\lambda, a}\right),\left(C_{\lambda, \mu}\right)$ and $\left(N_{\lambda, x_{0}}\right)$

For example, from Bratu's Theorem we have:

Gelfand Theorem. ([23]) For each $\lambda>0$ there exists $a^{*}(\lambda)$ such that:

(1) for $0<a<a^{*}(\lambda)$, the problem $\left(G_{\lambda, a}\right)$ has two solutions;

(2) the problem $\left(G_{\lambda}, a^{*}(\lambda)\right)$ has a unique solution;

(3) for $a>a^{*}(\lambda)$, the problem $\left(G_{\lambda, a}\right)$ has no solutions.

Proof. $y \in C^{2}(\mathbb{R})$ is a solution of $\left(B_{\lambda, b}\right)$ if and only if $y\left(x+\frac{b}{2}\right)$ is a solution of $\left(G_{\lambda, \frac{b}{2}}\right)$. See Remark 3.2(1) and Theorem 3.4.

From our remarks in Section 3, we also have: 
Theorem 4.1. (1) If $y \in C^{2}(\mathbb{R})$ is a solution of $\left(B_{\lambda, b}\right)$, then $y$ is a solution of $\left(N_{\lambda, \frac{b}{2}}\right)$.

(2) If $y \in C^{2}(\mathbb{R})$ is a solution of $\left(N_{\lambda, x_{0}}\right)$, then $y$ is a solution of $\left(B_{\lambda, 2 x_{0}}\right)$.

(3) If $y^{*}$ is the unique solution of $\left(B_{\lambda^{*}(b), b}\right)$ then there exists a unique $\mu^{*}>0$ such that $y^{*}$ is a solution of $\left(C_{\lambda^{*}(b), \mu^{*}}\right)$. If $0<\lambda<\lambda^{*}(b)$, then there exists $\mu_{1}<\mu^{*}<\mu_{2}$ such that if $y_{i}$ is the unique solution of $\left(C_{\lambda, \mu_{i}}\right)$, then the solution set of $\left(B_{\lambda, b}\right)$ is $\left\{y_{1}, y_{2}\right\}$. Moreover, $y_{1}<y^{*}<y_{2}$.

In what follow, we shall study the problem $\left(B_{\lambda, b}\right)$, where $0<\lambda<\lambda^{*}(b)$. From Theorem 4.1 it is clear that the problem $\left(B_{\lambda, b}\right)$ has a unique solution in the order interval $\left[0, y^{*}\right]$. On the other hand, the problem $\left(B_{\lambda, b}\right)$ is equivalent to the fixed point equation

$$
y(x)=\lambda \int_{0}^{b} G(x, s) e^{y(s)} d s, x \in[0, b] .
$$

Let $P_{\lambda}: C\left([0, b], \mathbb{R}_{+}\right) \rightarrow C\left([0, b], \mathbb{R}_{+}\right)$, be defined by

$$
P_{\lambda}(y)(x):=\lambda \int_{0}^{b} G(x, s) e^{y(s)} d s, x \in[0, b] .
$$

Notice that the operator $P_{\lambda}$ is completely continuous, increasing and $P_{\lambda}\left(\left[0, y^{*}\right]\right) \subset P_{\lambda}\left(\left[0, y^{*}\right]\right)$, for $0<\lambda<\lambda^{*}(b)$. By Theorem 2.4 we have the following result.

Theorem 4.2. For $0<\lambda<\lambda^{*}(b)$, the mapping $P_{\lambda}:\left[0, y^{*}\right] \rightarrow\left[0, y^{*}\right]$ defined by (4.2) is a Picard operator.

Proof. By Theorem 4.1, we get that $F_{P_{\lambda}}=\left\{y_{\lambda}\right\}$. By Theorem 2.4 we obtain

$$
P_{\lambda}^{n}(0) \rightarrow y_{\lambda} \text { as } n \rightarrow \infty \text { and } P_{\lambda}^{n}\left(y^{*}\right) \rightarrow y_{\lambda} \text { as } n \rightarrow \infty \text {. }
$$

Since $P_{\lambda}$ is increasing, if $y \in\left[0, y^{*}\right]$, then $P^{n}(0) \leq P^{n}(y) \leq P^{n}\left(y^{*}\right)$. This implies that $P^{n}(y) \rightarrow y_{\lambda}$ as $n \rightarrow \infty$.

On the other hand, since $\lambda>0$ and $b>0$, then

$$
\left\|P_{\lambda}(y)\right\|_{\infty} \leq \frac{\lambda b^{2}}{8} e^{\|y\|_{\infty}}, \text { for all } y \in C\left([0, b], \mathbb{R}_{+}\right) .
$$

Let $M>0$. If $\lambda$ and $b$ are such that $\frac{\lambda b^{2}}{8} e^{M} \leq M$, then the order interval $[0, M] \subset C\left([0, b], \mathbb{R}_{+}\right)$is invariant subset of $P_{\lambda}$. If, in addition, $\frac{\lambda b^{2}}{8} e^{M}<1$, then $P_{\lambda}:[0, M] \rightarrow[0, M]$ is a contraction. Thus, in terms of the Saturated Contraction Principle (see Theorem 2.3 or Theorem 1.1 in [49]) we can obtain more information with respect to the solution of $\left(B_{\lambda, b}\right)$ in $[0, M]$. We have the following result.

Theorem 4.3. Let us consider the problem $\left(B_{\lambda, b}\right)$. For $0<\lambda<\lambda^{*}(b)$ and $\lambda b^{2}<\frac{8}{e}$, take any $M \in] 0, \ln \frac{8}{\lambda b^{2}}[$. Then, the following conclusions hold:

(i) the problem $\left(B_{\lambda, b}\right)$ has a unique solution $y^{*}$ in the order interval $[0, M] \subset$ $C\left([0, b], \mathbb{R}_{+}\right) ;$ 
(ii) the sequence $\left(y_{n}\right)_{n \in \mathbb{N}}$ defined by

$$
y_{n+1}(x):=\lambda \int_{0}^{b} G(x, s) e^{y_{n}(s)} d s, x \in[0, b], n \in \mathbb{N},
$$

(where $y_{0}$ is arbitrary in the order interval $[0, M] \subset C\left([0, b], \mathbb{R}_{+}\right)$) converges to $y^{*}$;

(iii) for every $y$ from the order interval $[0, M] \subset C\left([0, b], \mathbb{R}_{+}\right)$we have

$$
\left\|y-y^{*}\right\|_{\infty} \leq \frac{1}{1-K}\left\|y-P_{\lambda} y\right\|_{\infty}
$$

where $P_{\lambda}(y)(x):=\lambda \int_{0}^{b} G(x, s) e^{y(s)} d s$ and $K:=\frac{\lambda b^{2}}{8} e^{M}$; that

(iv) if $\left(u_{n}\right)_{n \in \mathbb{N}}$ is a sequence in the order interval $[0, M] \subset C\left([0, b], \mathbb{R}_{+}\right)$such

$$
\left\|u_{n}-P_{\lambda} u_{n}\right\|_{\infty} \rightarrow 0 \text { as } n \rightarrow \infty
$$

then $u_{n} \rightarrow y^{*}$ as $n \rightarrow \infty$; that

(iv) if $\left(u_{n}\right)_{n \in \mathbb{N}}$ is a sequence in the order interval $[0, M] \subset C\left([0, b], \mathbb{R}_{+}\right)$such

$$
\left\|u_{n+1}-P_{\lambda} u_{n}\right\|_{\infty} \rightarrow 0 \text { as } n \rightarrow \infty
$$

then $u_{n} \rightarrow y^{*}$ as $n \rightarrow \infty$.

Proof. Consider the fixed point equation equation (4.1) and the operator $P_{\lambda}$ defined by (4.2). By the above assumptions, we have that

$$
\frac{\lambda b^{2}}{8} e^{M} \leq M \text { and } \frac{\lambda b^{2}}{8} e^{M}<1 .
$$

Thus, $P_{\lambda}:[0, M] \rightarrow[0, M]$ and it is a contraction. The rest of the conclusions follow from Theorem 1.1 in [49].

Remark 4.4. For a better understanding of this result it is useful to compare it with Theorem 1 in [29].

\section{Bratu-type problems}

From the above considerations (see Section 3) on Bratu's equation, we are motivated to adopt the following notions.

Let us consider the equation

$$
-y^{\prime \prime}=\lambda f(y)
$$

where $\lambda>0, f \in C^{2}(\mathbb{R})$ and $f^{(k)}(t)>0$, for all $t \in \mathbb{R}$ and $k \in\{0,1,2\}$. Since $f$ is locally Lipschitz, each Cauchy problem associated to $\left(E_{f, \lambda}\right)$ has a unique saturated solution $y \in C^{2}(] x_{-}, x_{+}[)$. We suppose that: $x_{-}=-\infty$ and $x_{+}=+\infty$.

By definition, the equation $\left(E_{f, \lambda}\right)$ is of Bratu-type if the above conditions are satisfied. In this case, we denote it by $(B T(f, \lambda))$.

As in Section 3, we have: 
Remark 5.1. If $y$ is a solution of $(B T(f, \lambda))$ then:

(1) $y$ is strictly concave function;

(2) the function $x \mapsto y(x+c), x \in \mathbb{R}$, is a solution of $(B T(f, \lambda))$, for all $c \in \mathbb{R}$;

(3) the function $x \mapsto y(-x+c), x \in \mathbb{R}$, is a solution of $(B T(f, \lambda))$, for all $c \in \mathbb{R}$;

(4) if $y^{\prime}\left(x_{0}\right)=0$, then $y\left(x_{0}-x\right)=y\left(x_{0}+x\right), \forall x \in \mathbb{R}$;

(5) if $y(0)=0, y^{\prime}\left(x_{0}\right)=0,0<x_{0}$, then $y\left(2 x_{0}\right)=0$;

(6) if $y(a)=0, y(b)=0, a<b$, then $y^{\prime}(a)>0, y^{\prime}(b)<0$ and $y(x)>0$, $\forall x \in] a, b[$.

By definition, we call the problem

$$
\left\{\begin{array}{l}
-y^{\prime \prime}=\lambda f(y) \\
y(0)=0, y(b)=0,0<b,
\end{array}\right.
$$

the Bratu-type problem. We denote it by $(B T(f, \lambda, b))$.

For the Bratu-type problem, we have the following result.

Theorem 5.2. For each $\lambda>0$, there exists $b^{*}(\lambda)>0$ such that:

(1) for $0<b<b^{*}(\lambda)$, the problem $(B T(f, \lambda, b))$ has two solutions;

(2) the problem $\left(B T\left(f, \lambda, b^{*}(\lambda)\right)\right)$ has a unique solution;

(3) for $b>b^{*}(\lambda)$, the problem $(B T(f, \lambda, b))$ has no solution.

For each $b>0$, there exists $\lambda^{*}(b)$ such that:

$\left(1^{\prime}\right)$ for $0<\lambda<\lambda^{*}(b)$, the problem $(B T(f, \lambda, b))$ has two solutions;

$\left(2^{\prime}\right)$ the problem $\left(B T\left(f, \lambda^{*}(b), b\right)\right)$ has a unique solution;

$\left(3^{\prime}\right)$ for $\lambda>\lambda^{*}(b)$, the problem $(B T(f, \lambda, b))$ has no solutions.

Proof. Let $g(t)=\int_{0}^{t} f(s) d s+1$. In terms of $g$, the Bratu-type problem takes the following form

$$
-y^{\prime \prime}=\lambda g^{\prime}(y), y(0)=0, y(b)=0 .
$$

If $y$ is a solution of this problem, then $y^{\prime}(0)=\mu>0$, and from

$$
-2 y^{\prime} y^{\prime \prime}=2 \lambda y^{\prime} g^{\prime}(y)
$$

we have that

$$
-y^{\prime 2}(x)+\mu^{2}=2 \lambda g(y(x))-2 \lambda, \forall x \in[0, b] .
$$

From now on, we follow Bratu's proof of his theorem.

\section{Other generalizations}

In this section, we shall consider the following boundary value problem with increasing nonlinearity (see $\$ 2.2$ ), denoted by $(B V P)$ :

$$
\left\{\begin{array}{l}
L(y):=-y^{\prime \prime}+p(x) y^{\prime}+q(x) y=f(x, y) \\
l_{1}(y)(a)=0, l_{2}(y)(b)=0
\end{array}\right.
$$

where $p, q \in C[a, b], f \in C\left([a, b] \times \mathbb{R}_{+}\right), f(x, t)>0$, for all $x \in[a, b], t \in \mathbb{R}_{+}$, and the interval $[a, b]$ is a strong uniqueness interval with respect to $\left(L, l_{1}, l_{2}\right)$. 
Let us denote

$$
S_{+}(B V P):=\left\{y \in C^{2}\left([a, b], \mathbb{R}_{+}\right) \mid y \text { is a solution of }(B V P)\right\} .
$$

The problem $(B V P)$ is equivalent to the fixed point equation (in $C\left([a, b], \mathbb{R}_{+}\right)$,

$$
y(x)=\int_{a}^{b} G(x, s) f(s, y(s)) d s, x \in[a, b],
$$

where $G(x, s)$ is the Green function corresponding to $\left(L, l_{1}, l_{2}\right)$.

Since $[a, b]$ is a strong uniqueness interval, hence $G(x, s) \geq 0, \forall x, s \in[a, b]$.

We consider the operator $P: C\left([a, b], \mathbb{R}_{+}\right) \rightarrow C\left([a, b], \mathbb{R}_{+}\right)$defined by

$$
P(y)(x):=\text { second part of }(I E) .
$$

It is clear that $S_{+}(B V P)=F_{P}$.

For the problem $(B V P)$, we have the following result:

Theorem 6.1. In addition, we suppose that:

(1) $f(x, \cdot): \mathbb{R}_{+} \rightarrow \mathbb{R}_{+}$is strictly increasing, $\forall x \in[a, b]$;

(2) the $(B V P)$ has a positive strict supersolution denoted by $\widehat{y}$.

In these conditions we have that:

(i) the ordered set $\left(S_{+}(B V P), \leq\right)$ has a minimum element $y_{\min }$ (see [39]);

(ii) $y_{\text {min }}=\lim _{n \rightarrow \infty} P^{n}(0)$, in $\left(C\left([a, b], \mathbb{R}_{+},\|\cdot\|_{\infty}\right)\right)$;

(iii) if $\lim P^{n}(\widehat{y})=y_{\min }$, then $\left.P\right|_{[0, \widehat{y}]}$ is $P O$.

Proof. First, we remark that the operator $P$ is completely continuous and strictly increasing. Moreover, 0 is a strict lower fixed point of $P$. Now the proof follows from Theorem 3.4.

Remark 6.2. If $q(x) \geq 0$, then Theorem 6.1 generalizes some results given in [31], [2], $[3],[43], \ldots$

Example 6.3. We consider the boundary value problem

$$
\begin{aligned}
& L(y)=\sum_{k=1}^{m} \lambda_{k} e^{u_{k} x}, x \in[a, b], \\
& l_{1}(y)(a)=0, l_{2}(y)(b)=0 .
\end{aligned}
$$

If $\lambda_{k}>0$ and $\mu_{k}>0$, then this problem satisfies the conditions of Theorem 6.1.

In what follows, we shall use Hadamard linearized technique to study some semilinear problems. Let us consider the following second-order linear differential equations

$$
L y:=-y^{\prime \prime}+p y^{\prime}+q y=0, \text { for } x \in[a, b], \text { where } p, q \in C[a, b]
$$

and

$$
L y:=-y^{\prime \prime}+p y^{\prime}+q y=f(x, y) \text {, for } x \in[a . b], p, q \in C[a, b] \text { and } f \in C([a, b] \times J),
$$


with $J \subset \mathbb{R}$ a nondegenerate interval.

If $y \in C^{2}[a, b]$ is a nontrivial solution of (6.1) such that $y(x) \geq 0$ for every $x \in[a, b]$, then $y(x)>0$ for every $x \in] a, b[$.

By this well-known property, we have the following lemma.

Lemma 6.4. We suppose that $\left.\frac{\partial f}{\partial y} \in C[a, b] \times J\right)$. If $y_{1}, y_{2} \in C^{2}[a, b]$ are two solutions of (6.2) such that $y_{1}(x) \leq y_{2}(x)$ for every $x \in[a, b]$, then $y_{1}(x)<y_{2}(x)$ for every $x \in] a, b[$.

Proof. We have that

$$
\begin{gathered}
L\left(y_{2}-y_{1}\right)(x)=f\left(x, y_{1}(x)\right)-f\left(x, y_{2}(x)\right)= \\
\int_{0}^{1} \frac{\partial f}{\partial y}\left(x, y_{1}(x)+t\left(y_{2}(x)-y_{1}(x)\right)\right) d t \cdot\left(y_{2}-y_{1}\right)(x),
\end{gathered}
$$

i.e., $u:=y_{2}-y_{1}$ is a solution of the linear equation

$$
L u(x)-Q\left(x, y_{1}(x), y_{2}(x)\right) u(x)=0,
$$

where $Q\left(x, y_{1}(x), y_{2}(x)\right):=\int_{0}^{1} \frac{\partial f}{\partial y}\left(x, y_{1}(x)+t\left(y_{2}(x)-y_{1}(x)\right)\right) d t$. Since $y_{1}(x) \leq y_{2}(x)$ for every $x \in[a, b]$, then $y_{1}(x)<y_{2}(x)$ for every $\left.x \in\right] a, b[$.

By the above lemma, we also have the following theorem.

Theorem 6.5. We suppose that the following assumptions hold:

(1) $\left.\frac{\partial f}{\partial y} \in C[a, b] \times J\right)$;

(2) $\frac{\partial f}{\partial y}(x, t)<0$ for every $x \in[a, b]$ and $t \in J$;

(3) the interval $[a, b]$ is a strong uniqueness interval corresponding to $\left(L, l_{1}, l_{2}\right)$, where $l_{1}(y)(a)=y(a)$ and $l_{2}(y)(b)=y(b)$.

Then, the boundary value problem

$$
\left\{\begin{array}{l}
L(y):=-y^{\prime \prime}+p(x) y^{\prime}+q(x) y=f(x, y), x \in[a, b] \\
y(a)=0, y(b)=0
\end{array}\right.
$$

has at most a solution.

Proof. Let $y_{1}, y_{2} \in C^{2}[a, b]$ are two solutions of (6.5) and $u:=y_{2}-y_{1}$. Then $u$ is a solution of the linear equation

$$
L u(x)-Q\left(x, y_{1}(x), y_{2}(x)\right) u(x)=0,
$$

where $Q\left(x, y_{1}(x), y_{2}(x)\right)$ was introduced in Lemma 6.4 and has the property that $Q\left(x, y_{1}(x), y_{2}(x)\right) u(x)<0$ for every $x \in[a, b]$. Thus $q(x)-Q\left(x, y_{1}(x), y_{2}(x)\right) u(x)>0$ for every $x \in[a, b]$. By (3) and Theorem 2.1 (ii), we get that $u:=0$.

Another result in the linear case is the following Sturm comparison theorem ([34], [46], [48], [24]).

Let $p, q_{1}, q_{2} \in C[a, b]$ with $q_{1}(x)<q_{2}(x)$ for every $x \in[a, b]$. Let $y$ be a nontrivial solution of

$$
L(y):=-y^{\prime \prime}+p(x) y^{\prime}+q_{1}(x) y=0, x \in[a, b]
$$

and $z$ be a notrivial solution of

$$
L(z):=-z^{\prime \prime}+p(x) z^{\prime}+q_{2}(x) z=0, x \in[a, b] .
$$


If $z(a)=z(b)=0$, then there exists $\left.x_{0} \in\right] a, b\left[\right.$ such that $y\left(x_{0}\right)=0$. By this result, we immediately obtain the following theorem.

Theorem 6.6. We consider the boundary value problem (6.5). We suppose that the following assumptions hold:

(1) $\left.\frac{\partial f}{\partial y} \in C[a, b] \times J\right)$;

(2) $\frac{\partial f}{\partial y}(x, \cdot): J \rightarrow \mathbb{R}$ is strictly increasing for every $t \in J$.

Then, each totally ordered subset of the solution set of (6.5) has at most two elements.

Proof. Let $y_{1} \leq y_{2} \leq y_{3}$ three solutions of (6.5). By Lemma 6.4 we get that $y_{1}<y_{2}<$ $y_{3}$ for every $\left.x \in\right] a, b\left[\right.$. Let $y:=y_{3}-y_{1}$ and $z:=y_{2}-y_{1}$. Then

$$
L y(x)-Q\left(x, y_{1}(x), y_{3}(x)\right) y(x)=0,
$$

and

$$
L z(x)-Q\left(x, y_{1}(x), y_{2}(x)\right) z(x)=0,
$$

for every $x \in[a, b]$. Since $\frac{\partial f}{\partial y}(x, \cdot)$ is strictly increasing for every $t \in J$ and $y(x)>z(x)$ for every $x \in] a, b[$, by Sturm comparison theorem we get that $y$ must change the sign in $] a, b[$. Since $y(x)>0$ for every $x \in] a, b[$, this is a contradiction to our initial assumption. The proof is complete.

Remark 6.7. For similar results to Theorem 6.6 see [44] (pp. 253-254) and the references therein. Another result for the boundary value problem (6.5) can be obtained by Theorem 2.4 .

\section{Numerical analysis of Bratu type problems}

We know that Bratu's problem (1.1) has the exact solution of the form:

$$
y(x)=-2 \log \left(\frac{\cosh \left[\left(x-\frac{1}{2}\right) \frac{\theta}{2}\right]}{\cosh \frac{\theta}{4}}\right),
$$

where $\theta$ is the solution of the equation

$$
\theta=\sqrt{2 \lambda} \cosh \frac{\theta}{4}
$$

To get numerical approximations for the solutions of the equation (7.1) we apply the Newton's method for finding the roots of the function

$$
\varphi(\theta)=\theta-\sqrt{2 \lambda} \cosh \frac{\theta}{4},
$$

defined by

$$
\theta_{n+1}=\theta_{n}-\frac{\varphi\left(\theta_{n}\right)}{\varphi^{\prime}\left(\theta_{n}\right)}
$$


with a starting value $\theta_{0} \in[a, b]$ chosen such that in $[a, b]$ equation (7.1) has only one solution and $\varphi^{\prime}\left(\theta_{0}\right) \neq 0$. It is clear that $\varphi \in C^{2}(\mathbb{R})$ is a concave function with a maximum in

$$
\theta^{*}(\lambda)=4 \operatorname{arcsinh}\left(\frac{4}{\sqrt{2 \lambda}}\right)
$$

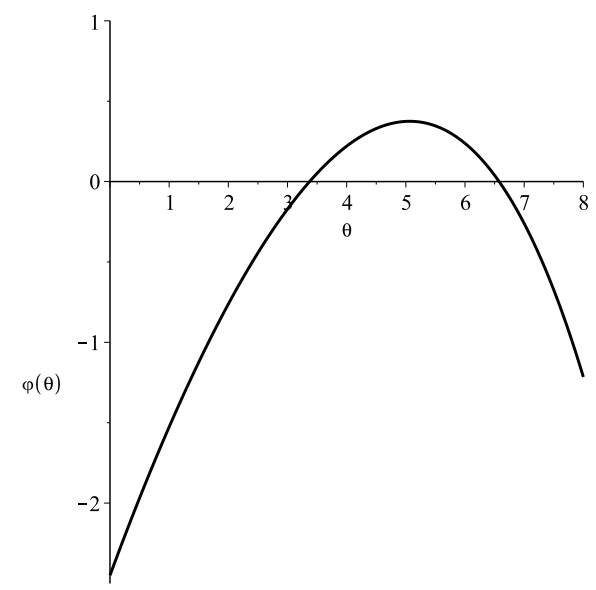

Figure 1. The graph of $\varphi(\theta)$ for $\lambda=3$.

The existence of the critical value $\lambda_{c}$ comes from the condition that, in order to have solutions for the equation (7.1), the maximum value $\varphi\left(\theta^{*}(\lambda)\right)$ should be nonnegative. Thus, $\lambda_{c}$ is obtained as a solution of

$$
\varphi\left(\theta^{*}(\lambda)\right)=0
$$

and, in the case of Bratu's problem (1.1), we have $\lambda_{c} \approx 3.513830719$.

If $0<\lambda<\lambda_{c}$, then $\varphi\left(\theta^{*}(\lambda)\right)>0$ and the equation has two solutions $\theta_{1}(\lambda), \theta_{2}(\lambda)$. Since $\varphi(0)<0$ and $\lim _{\theta \rightarrow+\infty} \varphi(\theta)=-\infty$ then $\theta_{1}(\lambda) \in\left(0, \theta^{*}(\lambda)\right)$ and $\theta_{2}(\lambda) \in\left(\theta^{*}(\lambda),+\infty\right)$. In order to get a numerical approximation of $\theta_{1}(\lambda)$, respectively, of $\theta_{2}(\lambda)$, we may choose as a starting value $\theta_{0} \in\left(0, \theta^{*}(\lambda)\right)$, respectively, $\theta_{0} \in\left(\theta^{*}(\lambda), \theta^{*}(\lambda)+\varepsilon\right)$ for some $\varepsilon>0$.

If $\lambda=\lambda_{c}$ then $\varphi\left(\theta^{*}\left(\lambda_{c}\right)\right)=0$, so $\theta^{*}\left(\lambda_{c}\right)$ is the unique solution of (7.1). This value can be obtained as the limit $\theta_{1}(\lambda)$ or $\theta_{2}(\lambda)$ when $\lambda \rightarrow \lambda_{c}$.

In the case of $\lambda=3$, we have

$$
\theta^{*}(3)=4 \operatorname{arcsinh}\left(\frac{2}{3} \sqrt{6}\right) \approx 5.065364187
$$

and the following iterations: 


\begin{tabular}{|c|c|c|}
\hline & $\theta_{0}=0$ & $\theta_{0}=10$ \\
\hline$\theta_{1}=$ & 2.4494897427831780982 & 8.1438003057516703864 \\
\hline$\theta_{2}=$ & 3.2377069463405279948 & 7.0734727359775392946 \\
\hline$\vdots$ & $\vdots$ & $\vdots$ \\
\hline$\theta_{10}=$ & 3.3735077642858915405 & 6.5765692592543752601 \\
\hline$\vdots$ & $\vdots$ & $\vdots$ \\
\hline$\theta_{19}=$ & 3.3735077642858915405 & 6.5765692592543752601 \\
\hline$\theta_{20}=$ & 3.3735077642858915405 & 6.5765692592543752601 \\
\hline
\end{tabular}

For different values of $\lambda<\lambda_{c}$, we obtain the following approximating values for $\theta_{1}(\lambda)$ and $\theta_{1}(\lambda)$ :

\begin{tabular}{|c|c|c|}
\hline$\lambda$ & $\theta_{1}(\lambda)$ & $\theta_{2}(\lambda)$ \\
\hline 1 & 1.5171645990507543685 & 10.938702772122106800 \\
\hline 2 & 2.3575510538774020426 & 8.5071995707130261296 \\
\hline 3 & 3.3735077642858915407 & 6.5765692592543752601 \\
\hline 3.513 & 4.7374700066634551382 & 4.8604846857553034188 \\
\hline 3.513830719125 & 4.7987137042679359281 & 4.7987154177935504693 \\
\hline
\end{tabular}

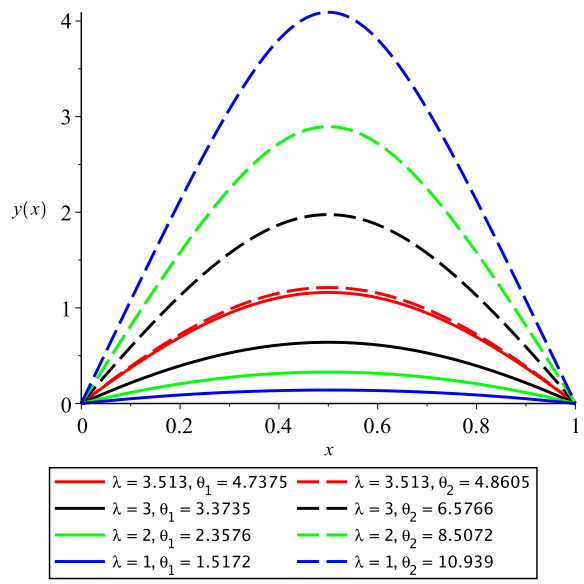

Figure 2. The graph of Bratu's problem solutions for different values of $\lambda$.

\section{References}

[1] Agarwal, R.P., Hodis, S., O'Regan, D., 500 Examples and Problems of Applied Differential Equations, Springer, 2019.

[2] Amann, H., Fixed point equations and nonlinear eigenvalue problems in ordered Banach spaces, SIAM Review, 18(1976), no. 4, 620-709. 
[3] Amann, H., Supersolutions, monotone iterations and stability, J. Diff. Eq., 21(1976), 363-377.

[4] Bailey, P., Shampine, L.F., Waltman, P., Existence and uniqueness of solution of the second order boundary value problem, Bull. Amer. Math. Soc., 72(1966), 96-98.

[5] Bailey, P., Shampine, L.F., Waltman, P., Nonlinear Two Point Boundary Value Problems, Acad. Press, New York, 1968.

[6] Bandle, C., Existence theorems, qualitative results and a priori bounds for a class of nonlinear Dirichlet problems, Arch. Rational Mech. Anal., 58(1975), 219-238.

[7] Ben-Romdhane, M., Temimi, H., Baccouch, M., An iterative finite difference method for approximating the two-branched solution of Bratu's problem, Appl. Numerical Math., 139(2019), 62-76.

[8] Bougoffa, L., Exact solutions of a generalized Bratu equation, Romanian J. Physics, 62(2017), article no. 110, 1-5.

[9] Boyd, J.P. Chebyshev polynomial expantions for simultaneous approximation of two branches of a function with application to the one-dimensional Bratu equation, Appl. Math. Comput., 142(2003), 189-200.

[10] Bratu, Gh., Sur certaines équations integrales nonlinéaire, C.R. Acad. Sci. Paris, 150(1910), 896-899.

[11] Bratu, Gh., Sur l'équation intégrale exponentielle, C.R. Acad. Sci. Paris, 152(1911), 1048-1050.

[12] Bratu, Gh., Sur les équations intégrales nonlinéaires, Bulletin de la Soc. Math. France, 42(1914), 113-142.

[13] Buckmire, R. Application of a Mickens finite-difference scheme to the cylindrical BratuGelfand problem, Numer. Methods Partial Diff. Equations, 20(2004), no. 3, 327-337.

[14] Caglar, H., Caglar, N., Özer, M., Anagnostopoulos, A.N., B-spline method for solving Bratu's problem, J. Comput. Math., 87(2010) no. 8, 1885-1891.

[15] Chandrasekhar, S., An Introduction to the Study of Stellar Structure, Dover Publ., New York, 1957.

[16] Cohen, N., Toledo Benavides, J.V., Exact solutions of Bratu and Liouville equations, Anais do CNMAC, 3(2010), 750-756.

[17] Feng, X., He, Y., Meng, J., Application of homotopy perturbation method to the Bratutype equations, Topological Meth. in Nonlinear Anal., 31(2008), 243-252.

[18] Foiaş, C., Gussi, G., Poenaru, V., Despre problema polilocală la ecuaţii diferenţiale liniare de ordinul al doilea, Buletin Ştiinţific, Sectiunea de Ştiinţe Matematice şi Fizice, 7(1955), no. 3, 699-721.

[19] Frank-Kamenetskii, D.A., Calculation of thermal explosion limits, Acta Phys., USSR, 10(1939), 365.

[20] Frank-Kamenetskii, D.A., Diffusion and Heat Exchange in Chemical Kinetics, Princeton Univ. Press, 1955.

[21] Fujita, H., On the nonlinear equations $\Delta u+e^{u}=0$ and $\frac{\partial v}{\partial t}=\Delta v+e^{v}$, Bull. Amer. Math. Soc., 75(1969), 132-135.

[22] Galaktionov, V.A., Vázquez, J.L., The problem of blow-up in nonlinear parabolic equations, Discrete and Continuous Dynamical Systems, 8(2002), no. 2, 399-433.

[23] Gelfand, I.M., Some problems in the theory of quasilinear equations, Amer. Math. Soc. Transl., 29(1963), 295-381 (Usp. Mat. Nauk, 14(1959), no. 2, 87-158). 
[24] Hartman, P., Ordinary Differential Equations, Wiley New York, 1964.

[25] Hirsch, M.W., The dynamical systems approach to differential equations, Bull. Amer. Math. Soc., 11(1984), no. 1, 1-64.

[26] Jacobsen, J., Schmitt, K., The Liouville-Bratu-Gelfand problem for radial operators, J. Diff. Eq., 184(2002), 283-298.

[27] Joseph, D., Lundgren, Quasilinear Dirichlet problems driven by positive source, Arch. Rational Mech. Anal., 49(1973), 241-269.

[28] Joseph, D., Sparrow, E.M., Nonlinear diffusion induced by nonlinear sources, Quarterly Appl. Math., 28(1970), no. 3, 327-342.

[29] Kafri, H.Q., Khuri, S.A., Bratu's problem: A novel approach using fixed point iterations and Green's functions, Computer Physics Communications, 198(2016), 97-104.

[30] Keller, H.B., Existence theory for two point boundary value problems, Bull. Amer. Math. Soc., 72(1966), 728-731.

[31] Keller, H.B., Cohen, D.S., Some positone problems suggested by nonlinear heat generation, J. Math. Mech., 16(1967), no. 12, 1361-1376.

[32] Khuri, S.A., Louhichi, I., A novel Ishikawa-Green's fixed point scheme for the solution of BVPs, Appl. Math. Letters, 82(2018), 50-57.

[33] Krasnoselskii, M.A., Positive Solutions of Operator Equations, Noordhof, 1964.

[34] Kreith, K. PDE generalization of the Sturm comparison theorem, Memoirs Amer. Math. Soc., 48(1984), no 298, 31-46.

[35] Laetsch, T., On the number of solutions of boundary value problems with convex nonlinearities, J. Math. Anal. Appl., 35(1971), 389-404.

[36] Lebovitz, N., Oscillation theory and the spectra of eigenvalues, in: Ordinary Differential Equations, http://people.cs.uchicago.edu/ lebovitz/odes.html

[37] Liouville, J., Sur l'équation aux différences partielles $\frac{d^{2} \log \lambda}{d u d v} \pm \lambda 2 a^{2}=0$, J. Math. Pures Appl., 18(1853), 71-72.

[38] Miller, K.S., Schiffer, M.M., Monotonic properties of the Green's function, Proc. Amer. Math. Soc., 3(1952), 848-956.

[39] Păcurar, M., Rus, I.A., Some remarks on the notations and terminology in the ordered set theory, Creat. Math. Inf., 27(2018), no. 2, 191-195.

[40] Petruşel, A., Rus, I.A., A class of functional-integral equations with applications to bilocal problem, in: Topics in Mathematical Analysis and Applications (T.M. Rassias, L. Toth - Eds.), Springer, 2014, 609-631.

[41] Picard, E., Mémoire sur la théorie des équations aux dérivées partielles et la méthode des approximations successives, J. Math. Pures Appl., 6(1890), 145-210.

[42] Picard, E., Sur l'application des méthodes d'approximations successives à l'étude de certaines équations différentielles ordinaires, J. Math. Pures Appl., 9(1893), 217-272.

[43] Picard, E., Sur certains exemples singuliers d'approximations successives, Comptes Rendus, 126(1898), 497-500.

[44] Piccinini, L.C., Stampacchia, G., Vidossich, G., Ordinary Differential Equations in $\mathbb{R}^{n}$, Springer, 1984.

[45] Polyamin, A.D., Zaitsev, V.F., Handbook of Ordinary Differential Equations: Exact Solutions, Methods and Problems, CRC Press, Boca Raton, 2018.

[46] Reid, W.T., Sturmian Theory for Ordinary Differential Equations, Springer, 1980. 
[47] Rus, I.A., Sur la positivité de la fonction de Green correspondante au problem bilocal, Glasnik Mat., 5(1970), 251-257.

[48] Rus, I.A., Ecuaţii diferenţiale, ecuaţii integrale şi sisteme dinamice, Transilvania Press Cluj-napoca, 1996.

[49] Rus, I.A., Some variants of contraction principle, generalization and applications, Stud. Univ. Babeş-Bolyai Math., 61(2016), no. 3, 343-358.

[50] Rus, I.A., Petruşel, A., Petruşel, G., Fixed Point Theory, Cluj Univ. Press, Cluj-Napoca, 2008.

[51] Syam, M.I., Hamdan, A., An efficient method for solving Bratu equations, Appl. Math. Comput., 176(2006), 704-713.

[52] Wazwaz, A.M., Adomian decomposition method for a reliable treatment of the Bratu-type equations, Appl. Math. Comput., 166(2005), 652-663.

Adrian Petruşel

Babeş-Bolyai University, Faculty of Mathematics and Computer Science

Kogălniceanu Str. no. 1, 400084 Cluj-Napoca, Romania

and

Academy of Romanian Scientists Bucharest, Romania

e-mail: petrusel@math.ubbcluj.ro

Ioan A. Rus

Babeş-Bolyai University, Faculty of Mathematics and Computer Science

Kogălniceanu Str. no. 1, 400084 Cluj-Napoca, Romania

e-mail: iarus@math.ubbcluj.ro

Marcel Adrian Şerban

Babeş-Bolyai University, Faculty of Mathematics and Computer Science

Kogălniceanu Str. no. 1, 400084 Cluj-Napoca, Romania

e-mail: mserban@math.ubbcluj.ro 